\title{
A COMPARATIVE DRUG UTILIZATION STUDY OF DEPRESSION PATIENTS BETWEEN TERTIARY CARE TEACHING HOSPITAL AND PRIVATE PRACTITIONERS OF DEHRADUN CITY, UTTARAKHAND
}

\author{
${ }^{1}$ Dr. Shaktibala Dutta, ${ }^{2}$ Dr. Vijay Kaul, ${ }^{3 *}$ Dr. Mirza Atif Beg, ${ }^{4}$ Dr. Nand Kishore Singh, ${ }^{5}$ Dr. Srihari Dutta, \\ ${ }^{6}$ Dr. Shalu Bawa, ${ }^{7}$ Dr. Mohammad Anjoom, ${ }^{8}$ Dr. Saubhagya Sindhu, ${ }^{9}$ Dr. Ankita Negi \\ ${ }^{1}$ Department of Pharmacology, SGRRIM\&HS, Patel Nagar, Dehradun, India \\ ${ }^{2}$ Department of Psychiatry, Nepalganj Medical College, Nepal \\ ${ }^{3}$ Department of Pharmacology, SGRRIM\&HS, Patel Nagar, Dehradun, India \\ ${ }^{4}$ Department of Psychiatry, SGRRIM\&HS, Patel Nagar, Dehradun, India \\ ${ }^{5}$ Immunization Health Specialist, Unicef, New Delhi, India Country Office India. \\ ${ }^{6}$ Department of Pharmacology, SGRRIM\&HS, Patel Nagar, Dehradun, India \\ ${ }^{7}$ Department of Pharmacology, SGRRIM\&HS, Patel Nagar, Dehradun, India \\ ${ }^{8}$ Department of Pharmacology, SGRRIM\&HS, Patel Nagar, Dehradun, India \\ ${ }^{9}$ Department of Pharmacology, SGRRIM\&HS, Patel Nagar, Dehradun, India \\ *Corresponding Author's Email: mabeg1997@gmail.com
}

Received 22 Jan 2015; Review Completed 19 Feb 2015; Accepted 13 March 2015, Available online 15 march 2015

\begin{abstract}
Background: Drug utilization studies are beneficial in clinical practice for rational prescribing, as there is continuous proliferation of new drugs and the increasing recognition of their delayed adverse effects. Depression is an important global public health problem thus making such studies a must for minimizing the medication errors.

Aim and objectives: Present study audits the prescribing pattern of psychiatric private practitioners (PPs) vs psychiatric practitioners of tertiary care teaching hospital (TCTH) at Dehradun, Uttarakhand.

Materials and methods: A total of 371 prescriptions were analysed, 196 were collected from PPs and 175 from TCTH at Dehradun. They were analysed using WHO drug use indicators and National List of Essential Medicines (NLEM-2013).

Results: Anxiolytics 150(25.25\%) were most commonly prescribed by the PPs followed by antidepressants $120(20.20 \%)$ and antipsychotics $111(18.48 \%)$, whereas antidepressants $105(27.78 \%)$ was the major drug group prescribed by TCTH followed by anxiolytics $102(26.98 \%)$ and antipsychotics $37(9.79 \%)$. Tricyclic antidepressants(TCAs) were most frequently prescribed antidepressants followed by selective serotonin reuptake inhibitors(SSRIs) at TCTH whereas SSRIs were the major antidepressants prescribed by PPs. Anxiolytics and anticholinergics co-prescription with antidepressants and antipsychotics respectively, was a common observation among PPs and TCTH. Analysis of prescription revealed average number of drugs prescribed per prescription in teaching hospital is 2.16 vs 3.03 among the private practitioners. 18.25\% Fixed drug combinations(FDCs) were prescribed by TCTH while $0 \%$ were prescribed by PPs. $55.39 \%$ and $55.56 \%$ drugs were prescribed from the NLEM-2013 by the PPs and the TCTH respectively.

Conclusion: There were lesser number of drugs prescribed per prescription and more frequent use of drug combinations at TCTH while no drug combinations and greater number of drugs per prescription were prescribed by PPs. Newer class of drugs were more frequently prescribed by PPs.

Key words: Drug utilization studies, antidepressants, antipsychotics, anxiolytics, Fixed drug combinations.
\end{abstract}

\section{INTRODUCTION}

The World Health Organisation (WHO) defines drug utilization as the marketing, distribution, prescription and the use of drugs in a society, with special emphasis on the resulting medical, social and economic consequences ${ }^{1}$. Rational drug prescribing is the use of the least number of drugs to obtain the best possible effect in the shortest period and at a reasonable cost ${ }^{2}$. Irrational prescribing and disparity between the prescription and the consumption of medicines may offset the benefits. The recent proliferation of new 
drugs and the increasing recognition of delayed adverse effects have stimulated interest in the prescribing patterns of physicians. Depression is an important global public health problem and is a major cause of disability and premature death ${ }^{3}$. With the advancement of new drug research and better research outcome, psychotropic drug prescribing patterns have changed globally over the last few years and limited data is available explaining the drug utilization pattern and awareness among the psychiatric practitioners. Therefore the objective of the present study was to observe the prescribing pattern of drugs among psychiatrists and to compare the drug utilization pattern between a tertiary care teaching hospital and private psychiatric practitioners at Dehradun, Uttarakhand.

\section{MATERIALS AND METHOD}

Prescriptions were collected randomly from private psychiatric practitioners (PPs) as well as a Tertiary Care Teaching Hospital (TCTH) at Dehradun. Prescriptions were collected from the OPD during first two hours while patients were making their visits. Prescriptions were entered in a preformed proforma and were analysed on the basis of WHO drug use indicators and National List of Essential Medicines (NLEM-2013).

\section{RESULT}

A total number of 371 prescriptions were analysed, of which 196 were from PPs and 175 from TCTH. The mean age of patients from TCTH was $38.22 \pm 1.03$ years while that from PPs was $40.41 \pm 1.34$ years. Out of total 196 patients from PPs, 106 (54.08\%) were females and $90(45.92 \%)$ were males. Out of total 175 patients from TCTH, $99(56.57 \%)$ were females and 76 $(43.43 \%)$ were males. Majority of patients were married $(76 \%, 52.04 \%)$ and were housewives $(45.14 \%$, $28.06 \%$ ) in TCTH and PPs data respectively (Table 1).

Table 1: Comparison of Demographic profile

\begin{tabular}{|c|c|c|}
\hline Parameters & $\begin{array}{c}\text { TCTH } \\
(\mathbf{n = 1 7 5})\end{array}$ & PPs (n=196) \\
\hline Mean age & $38.22 \pm 1.03$ & $40.41 \pm 1.34$ \\
\hline Age in years & & \\
\hline$<18$ & $4(2.29 \%)$ & $15(7.65 \%)$ \\
\hline $18-30$ & $49(28.0 \%)$ & $49(25.0 \%)$ \\
\hline $31-50$ & $93(53.14 \%)$ & $79(40.31 \%)$ \\
\hline $51-70$ & $24(13.71 \%)$ & $46(23.47 \%)$ \\
\hline$>70$ & $5(2.86 \%)$ & $7(3.57 \%)$ \\
\hline Female:Male & $1.30: 1$ & $1.17: 1$ \\
\hline Married:Unmarried & $3.17: 1$ & $1.58: 1$ \\
\hline Occupation & & \\
\hline Housewife & $79(45.14 \%)$ & $55(28.06 \%)$ \\
\hline Government job & $15(8.57 \%)$ & $38(19.39 \%)$ \\
\hline Businessman & $39(22.29 \%)$ & $42(21.43 \%)$ \\
\hline Student & $27(15.43 \%)$ & $33(16.84 \%)$ \\
\hline Retired & $5(2.86 \%)$ & $10(5.10 \%)$ \\
\hline Unemployed & $10(5.71 \%)$ & $18(9.18 \%)$ \\
\hline
\end{tabular}

Table 2: Drug prescribing pattern

\begin{tabular}{|c|c|c|}
\hline Drug group & $\begin{array}{c}\text { TCTH } \\
(\mathbf{n = 3 7 8})\end{array}$ & PPs (n=594) \\
\hline Antianxiety & $102(26.98 \%)$ & $150(25.25 \%)$ \\
\hline Antidepressants & $105(27.78 \%)$ & $120(20.20 \%)$ \\
\hline Antipsychotics & $37(9.79 \%)$ & $111(18.48 \%)$ \\
\hline Antiepileptic & $30(7.94 \%)$ & $92(15.39 \%)$ \\
\hline Anticholinergics & $11(2.91 \%)$ & $6(1.01 \%)$ \\
\hline Others & $93(24.60 \%)$ & $118(19.67 \%)$ \\
\hline
\end{tabular}

Among the different categories of drugs prescribed, anxiolytics $150 \quad(25.25 \%)$ were most commonly prescribed drug group by the PPs followed by antidepressants $120(20.20 \%)$ and antipsychotics 111 (18.48\%), whereas antidepressants 105 (27.78\%) was the major drug group prescribed by TCTH followed by anxiolytics $102(26.98 \%)$ and antipsychotics 37 (9.79\%) (Table 2).

Among 120 antidepressants prescribed by PPs, Venlafaxine $37(30.83 \%)$ was the most common one, followed by mirtazapine $30(25 \%)$, fluoxetine 21 $(17.5 \%)$, escitalopram $19(15.83 \%)$, paroxetine 8 $(6.67 \%)$, duloxetine $3(2.5 \%)$ and fluvoxamine 2 (1.67\%). Among 105 antidepressants prescribed by TCTH, Amitriptyline 26 (24.76\%) constituted major group followed by Escitalopram 24 (22.86\%), venlafaxine $20(19.04 \%)$, fluoxetine $12(11.43 \%)$, nortryptyline $7(6.67 \%)$, duloxetine $7(6.67 \%)$ and others (Paroxetine, Fluvoxamine, Mirtazapine) (Table $3)$.

Out of total 150 anxiolytics prescribed by PPs, alprazolam $89(59.33 \%)$ was most common drug prescribed followed by lorazepam $24(16 \%)$, zolpidem $22(14.67 \%)$ and others (Propranolol, Clonazepam, Etiozolam, Ramelteon, Diazepam). Among 102 anxiolytics prescribed by TCTH, propranolol 44 $(43.14 \%)$ was most commonly prescribed followed by diazepam 35 (34.31\%), clonazepam 18 (17.65\%), lorazepam $4(3.92 \%)$ and etiozolam $1(0.98 \%)$ (Table 4).

Table 3: Most commonly prescribed antidepressant drugs

\begin{tabular}{|c|c|c|}
\hline Drugs & TCTH(n=105) & PPs(n=120) \\
\hline Fluoxetine & $12(11.43 \%)$ & $21(17.5 \%)$ \\
\hline Amitriptyline & $26(24.76 \%)$ & - \\
\hline Escitalopram & $24(22.86 \%)$ & $19(15.83 \%)$ \\
\hline Paroxetine & $1(0.95 \%)$ & $8(6.67 \%)$ \\
\hline Fluvoxamine & $3(2.86 \%)$ & $2(1.67 \%)$ \\
\hline Nortryptyline & $7(6.67 \%)$ & - \\
\hline Venlafaxine & $20(19.04 \%)$ & $37(30.83 \%)$ \\
\hline Duloxetine & $7(6.67 \%)$ & $3(2.5 \%)$ \\
\hline Mirtazapine & $5(4.76 \%)$ & $30(25 \%)$ \\
\hline
\end{tabular}


Table 4: Prescribing trend of anti-anxiety drugs

\begin{tabular}{|c|c|c|}
\hline Drugs & TCTH(n=102) & PPs(n=150) \\
\hline Alprazolam & - & $89(59.33 \%)$ \\
\hline Lorazepam & $4(3.92 \%)$ & $24(16 \%)$ \\
\hline Zolpidem & - & $22(14.67 \%)$ \\
\hline Propranolol & $44(43.14 \%)$ & $5(3.33 \%)$ \\
\hline Clonazepam & $18(17.65 \%)$ & $5(3.33 \%)$ \\
\hline Etiozolam & $1(0.98 \%)$ & $2(1.33 \%)$ \\
\hline Ramelteon & - & $2(1.33 \%)$ \\
\hline Diazepam & $35(34.31 \%)$ & $1(0.68 \%)$ \\
\hline
\end{tabular}

111 antipsychotics were prescribed by PPs compared to 37 prescribed by TCTH. Olanzapine 47 (42.34\%) was most commonly prescribed followed by Risperidone 27 (24.32\%), Haloperidol 14 (12.61\%), Aripiprazole 11 (9.91\%), Quetiapine 7 (6.31\%), Trifluoperazine $3(2.71 \%)$ and Amisulpiride $2(1.80 \%)$. Among 37 antipsychotics prescribed by TCTH, Risperidone 20 (54.05\%) was the most common drug prescribed followed by olanzapine 10 (27.03\%), Levosulpiride $4(10.81 \%)$, Amisulpiride $2(5.41 \%)$ and Quetiapine 1 (2.70\%) (Table 5).

Table 5: Prescribing trend of antipsychotics

\begin{tabular}{|c|c|c|}
\hline Drugs & TCTH(n=37) & PPs(n=111) \\
\hline Olanzapine & $10(27.03 \%)$ & $47(42.34 \%)$ \\
\hline Risperidone & $20(54.05 \%)$ & $27(24.32 \%)$ \\
\hline Aripiprazole & - & $11(9.91 \%)$ \\
\hline Quetiapine & $1(2.70 \%)$ & $7(6.31 \%)$ \\
\hline Amisulpiride & $2(5.41 \%)$ & $2(1.80 \%)$ \\
\hline Levosulpiride & $4(10.81 \%)$ & - \\
\hline Haloperidol & - & $14(12.61 \%)$ \\
\hline Trifluoperazine & - & $3(2.71 \%)$ \\
\hline
\end{tabular}

Among the antiepileptics/mood stabilizers prescribed, 92 were prescribed by PPs of which which, Divalproex sodium $41(44.37 \%)$, Lamotrigine $26(28.26 \%)$ and Lithium Carbonate 12 (13.04\%) were frequently prescribed, while, a total of 30 drugs were prescribed by TCTH of which Divalproex sodium 25 (83.33\%), Pregabalin 3 (10\%), Lamotrigine 1 (3.33\%), and Topiramate $1(3.33 \%)$, were commonly prescribed (Table 6).

Table 6: Prescribing trend of antiepileptics/mood stabilizers

\begin{tabular}{|c|c|c|}
\hline Drugs & TCTH(n=30) & PPs(n=92) \\
\hline Divalproex sodium & $25(83.33 \%)$ & $41(44.37 \%)$ \\
\hline Pregabalin & $3(10.0 \%)$ & - \\
\hline Lamotrigine & $1(3.33 \%)$ & $26(28.26 \%)$ \\
\hline Lithium Carbonate & - & $12(13.04 \%)$ \\
\hline Levetiracetam & - & $6(6.32 \%)$ \\
\hline Oxcarbazepine & - & $6(6.32 \%)$ \\
\hline Topiramate & $1(3.33 \%)$ & $1(1.69 \%)$ \\
\hline
\end{tabular}

The average number of drugs prescribed per prescription by PPs was 3.03, while that by TCTH was 2.16. $100 \%$ drugs were prescribed by brand names. $18.25 \%$ Fixed drug combinations (FDCs) were prescribed by TCTH while $0 \%$ were prescribed by PPs. $55.39 \%$ and $55.56 \%$ drugs were prescribed from the NLEM-2013 by the PPs and the TCTH respectively (Table 8).

Table 7: Most commonly prescribed drugs

\begin{tabular}{|c|c|c|}
\hline Drug group & TCTH (n=378) & PPs (n=594) \\
\hline \multirow{2}{*}{ Antianxiety } & Propranolol & Alprazolam \\
\cline { 2 - 3 } & Diazepam & Lorazepam \\
\hline \multirow{2}{*}{ Antidepressants } & Amitriptyline & Venlafaxine \\
\cline { 2 - 3 } & Escitalopram & Mirtazapine \\
\cline { 2 - 3 } & Venlafaxine & Fluoxetine \\
\hline Antipsychotics & Risperidone & Olanzapine \\
\cline { 2 - 3 } & Olanzapine & Risperidone \\
\cline { 2 - 3 } & Levosulpiride & Haloperidol \\
\hline Antiepileptic & Divalproex sodium & Divalproex sodium \\
\cline { 2 - 3 } & Pregabalin & Lamotrigine \\
\hline Anticholinergics & Trihexiphenidyl & Trihexiphenidyl \\
\hline Others & Multivitamins & Calcium salts \\
\cline { 2 - 3 } & Non-Steroidal Anti- & Multivitamins \\
\cline { 2 - 3 } & Inflammatory Drugs (NSAIDs) & \\
\hline
\end{tabular}

Table 8: WHO recommended prescribing indicators

\begin{tabular}{|c|c|c|}
\hline Parameters & TCTH & PPs \\
\hline Average number of drugs/prescription & 2.16 & 3.03 \\
\hline Percentage of the drugs prescribed by generic name & $0 \%$ & $0 \%$ \\
\hline Percentage of fixed drug combinations (FDCs) & $18.25 \%$ & $0 \%$ \\
\hline Percentage of the drugs prescribed from NLEM 2013 & $55.56 \%$ & $55.39 \%$ \\
\hline
\end{tabular}




\section{DISCUSSION}

Depression is an important global public health problem and is a major cause of disability and premature death ${ }^{3}$. Drug utilization studies are pointers to prescribing behaviour of clinicians and help in improving it. A prescription therefore may be taken as a reflection of the physician's attitude, whether a private practitioner or working in a tertiary care teaching hospital, towards the disease and the role of the drug in its treatment providing an insight into the nature of the health care delivery system.

Out of total of 371 prescriptions analysed, 196 from PPs and 175 from TCTH, female patients formed the majority and is consistent with studies by Grover et al and Avanthi et al ${ }^{4,5}$. Majority of patients in both TCTH and PPs, were in the age group range of 30-51 years in both sexes, which is similar to studies by Dutta et al and Trivedi et al 6, 7 . Depression was common among housewives in both TCTH and PPs and is comparable to previous study ${ }^{5}$.

Among the different categories of drugs prescribed at TCTH and by PPs, anxiolytics, anti-depressants and antipsychotics were the most commonly drug groups prescribed which is consistent with previous studies ${ }^{4,7}$. Out of total antidepressants prescribed by PPs, SSRIs (Selective Serotonin Reuptake Inhibitors) were the most common followed by SNRIs (Serotonin Norepinephrine Reuptake Inhibitors) and atypical antidepressants whereas TCAs (Tricyclic Antidepressants) in addition to the above groups were prescribed at TCTH. The prescribing pattern of antidepressants is consistent with previous studies ${ }^{8,9}$. This difference in prescribing pattern was observed probably due to the fact that patients visiting ТCTH were from middle to lower socioeconomic class and the TCAs are relatively cheaper compared to SSRIs. Better tolerability, combined with their mild adverse effects, accounts for the popularity of SSRIs as the most widely prescribed anti-depressants 5, 10. Current treatment guidelines mostly recommend use of SSRIs as the first line agents in patients of depression ${ }^{11}$.

Anxiety symptoms are co-morbid with depression and benzodiazepines, among anxiolytics, were most commonly prescribed at TCTH and by PPs, consistent with previous studies ${ }^{4,7}$. Newer non-benzodiazepines drugs, Zolpidem and Ramelteon were prescribed by PPs depicting the increase in awareness and the readiness, to use new drugs, among the psychiatric PPs. Another reason for higher rate of prescription of benzodiazepine by PPs, could be anticipated worsening of anxiety which is commonly seen with the use of SSRIs.

Atypical antipsychotics were frequently prescribed compared to the typical or classical antipsychotics at TCTH and by PPs, consistent with previous studies ${ }^{6,7}$, 9, 12 . Atypical antipsychotics are now rated as first line agents since they have low propensity to cause extrapyramidal side effects, efficacy against refractory cases, better tolerance, low relapse rate, and safer adverse effect profile ${ }^{12}$. Trihexyphenidyl was the most common anticholinergic prescribed both at TCTH and by PPs, similar to previous studies ${ }^{6,8,9}$. Similar observation was observed in a study by Ren et al. where when anticholinergic agents were used concomitantly with atypical antipsychotics; patients tend to stay on target drug significantly longer than those who did not use any anticholinergic agents ${ }^{13}$.

The average number of drugs per prescription is important in psychiatry as polypharmacy and misuse of psychotherapeutic drugs is common ${ }^{5}$. The average number of drugs per prescription prescribed by PPs was 3.03 while at TCTH was 2.16 , comparable to studies by Lahon et al and Memon et al where 2.32 and 2.72 drugs were prescribed per prescription respectively 2,14 . Although irrational polypharmacy occurs too frequently but in many instances it is necessary to manage the patient with multiple medications and that makes rational polypharmacy. No FDCs were prescribed by PPs but $18.25 \%$ FDCs were prescribed at TCTH, which can be compared to previous studies, in one wherein no FDCs were prescribed while in other 91\% FDCs were prescribed 2, 5. Almost equal number of drugs was prescribed from the NEML-2013, 55.39\% and 55.56\% by PPs and at TCTH respectively, which is higher compared to previous study ${ }^{2} .100 \%$ drugs were prescribed by their brand names by both PPs and at TCTH, which is not in accordance with the WHO guidelines, as rational prescribing requires generic prescription, suggesting popularity of brands and influence of pharmaceutical companies amongst the psychiatrists.

\section{CONCLUSION}

Studying and analysing the prescription patterns seeks to monitor, evaluate and help the physicians in understanding how the available drugs can be best put to use practically. They suggest, if necessary, modifications in prescribing patterns so as to make medical care rational and our study was a step in that direction. In the present study, it was difficult to come to a specific conclusion on rational prescribing in the prescriptions collected from the PPs and TCTH. There were lesser number of drugs prescribed per prescription and more frequent use of drug combinations at TCTH while no drug combinations and greater number of drugs per prescription were prescribed by PPs. Newer class of drugs were being increasingly prescribed by PPs, SSRIs as antidepressants and non-benzodiazepine anxiolytics, zolpidem and ramelteon. This study may help to identify the problems involved in therapeutic decision making, among the psychiatric practitioners based on which future research studies could be contemplated to promote rational prescribing. 


\section{REFERENCES}

1. World Health Organisation. Introduction to drug utilisation research. Oslo: World Health Organisation, 2003.

2. Lahon K, Shetty HM, Paramel A, Sharma G. A Retrospective Drug Utilization Study of Antidepressants in the Psychiatric Unit of a Tertiary Care Hospital. Journal of Clinical and Diagnostic Research 2011; 5(5):1069-75.

3. Akiskal, H.S. Mood disorders: Historical introduction and conceptual overview. In: Kaplan and Sadock's comprehensive textbook of psychiatry. 8th edition. Lippincott Williams \& Wilkins: Philadelphia; 2005:1559-75.

4. Grover S, Avasth A, Kalita K, Dalal PK, Rao GP, Chadda RK, et al. IPS multicentric study: antidepressant prescription patterns. Indian J Psychiatry 2013; 55(1):41-5.

5. Avanthi E, Somashekar HE, Kumar P, Sushma HK, Sudarshan CY et al. Prescribing pattern of antidepressants in psychiatric unit of a tertiary care hospital. Int J Basic Clin Pharmacol 2014; 3(4):667-70.

6. Dutta SB, Beg MA, Sindhu S, Singh NK. Role of pharmacoepidemiology in psychopharmacology: a study in psychiatric out-patient department of a tertiary care teaching hospital at Dehradun, Uttarakhand. Int $\mathrm{J}$ Basic Clin Pharmacol 2014;3(4):637-43.

7. Trivedi JK, Dhyani M, Sareen H, Yadav VS, Rai SB. Antidepressant drug prescription pattern for depression at a tertiary health care centre of Northern India. Med Pract Rev 2010; 1(2):16-8.
8. Dutta S, Beg MA, Kaul V, Dutta SH, Dhasmana DC. Psychotropic drug utilization study in psychiatric OPD of a tertiary care teaching hospital in Dehradun, Uttarakhand. J Adv Res Biol Sci 2013; 5(4):386-91.

9. Piparva KG, Parmar DM, Singh AP, Gajera MV, Trivedi HR Drug utilization study of psychotropic drugs in outdoor patients in a teaching hospital. Indian J Psychol Med 2011; 33(1):54-8.

10. Battista CD. Antidepressant agents. In: Katzung BG, Masters SB, Trevor AJ, editors. Basic and Clinical Pharmacology. 11th Edition. New York: McGraw Hill; 2012:p.509-30.

11. American Psychiatric association (APA). Practice guidelines for the treatment of patients with major depressive disorder. 3rd ed. Arlington (VA): American Psychiatric association (APA) 2010. 152p.(1170 references)

12. Dhasmana DC, Rawat Y, Mishra KC. What is so atypical about atypical antipsychotic? Indian J Pharmacol. 2003;35:322-4.

13. Ren XS, Huang YH, Lee AF, Miller DR, Qian S, Kazis L. Adjunctive use of atypical antipsychotics and anticholinergic drugs among patients with schizophrenia. J Clin Pharm Ther.2005; 30(1):65-71.

14. Memom A, Patel K. drug use pattern of antidepressant agents in psychiatric patients-a prospective study. NHL Journal of Medical Sciences 2013; 2(2):33-6. 